\title{
Activités
}

15-1 | 2018

Mettre en scène l'activité : les nouvelles figures de la simulation

\section{Développer les capabilités des patients pour coproduire un soin sûr et efficace}

Enhancing patients' capabilities to coproduce safe and effective care

\section{Adeline Pernet, Vanina Mollo et Pierre Falzon}

\section{(2) OpenEdition}

\section{Journals}

Édition électronique

URL : http://journals.openedition.org/activites/3065

DOI : 10.4000/activites.3065

ISSN : 1765-2723

Éditeur

ARPACT - Association Recherches et Pratiques sur les ACTivités

Référence électronique

Adeline Pernet, Vanina Mollo et Pierre Falzon, « Développer les capabilités des patients pour coproduire un soin sûr et efficace », Activités [En ligne], 15-1 | 2018, mis en ligne le 15 avril 2018 , consulté le 19 avril 2019. URL : http://journals.openedition.org/activites/3065 ; DOI : 10.4000/ activites.3065

Ce document a été généré automatiquement le 19 avril 2019

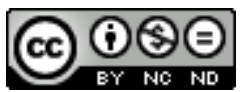

Activités est mis à disposition selon les termes de la licence Creative Commons Attribution - Pas d'Utilisation Commerciale - Pas de Modification 4.0 International. 


\section{Développer les capabilités des patients pour coproduire un soin sûr et efficace}

Enhancing patients' capabilities to coproduce safe and effective care

Adeline Pernet, Vanina Mollo et Pierre Falzon

\section{NOTE DE L'ÉDITEUR}

Article soumis le 26/01/2016, accepté le 24/09/2017

Les auteurs remercient :

L'Institut National du Cancer (INCa) pour avoir financé ce travail de recherche (Empowering patient in Patient safety: EMPATHY);

Tous les professionnels et patients qui ont accepté de participer à cette étude ;

Les reviewers pour leur lecture attentive et leurs critiques constructives.

\section{Introduction}

1 La sécurité des soins est un enjeu majeur des systèmes de santé (WHO, 2013). Elle renvoie évidemment à la sécurité des patients, qui consiste à être à l'abri des blessures accidentelles (Kohn, Corrigan, \& Donaldson, 1999), et désigne de façon générale l'aptitude d'un système à accomplir une mission particulière : celle d'éviter autant que possible l'occurrence d'événements indésirables (Neboit, Cuny, Fadier, \& Ho, 1990 ; Hollnagel, 2010). Son but est de produire un soin sûr et efficace (Pernet, 2013).

2 La radiothérapie est l'une des premières spécialités médicales à avoir intégré dans son fonctionnement une conscience du risque, suite à différents accidents comme ceux d'Épinal, de Toulouse ou de Grenoble pour les plus emblématiques (Mollo, \& al., 2011). 
Comme souligné par Michel Bourguignon, commissaire à l'Autorité de Sûreté Nucléaire (ASN, 2009), les efforts déployés pour améliorer la prévention des accidents en radiothérapie ont porté leurs fruits (renouvellement des appareils de traitement, renforcement des effectifs, organisation du retour d'expérience, etc.); mais la situation reste fragile. Il est donc important de rechercher d'autres voies d'amélioration de la qualité et de la sécurité des soins en radiothérapie. L'une d'entre elles consiste à renforcer la coopération des patients dans la production d'un soin sûr et efficace. Même si l'implication des patients est reconnue comme un moyen d'améliorer la qualité et la sécurité des soins (Hall, 2007 ; Coulter, \& Ellins, 2007), elle demeure peu étudiée à ce jour (Jorm, Dunbar, Sudano, \& Travaglia, 2009 ; Vincent, \& Davis, 2012), notamment en France. Pourtant, plusieurs arguments plaident en faveur de son développement.

D'une part, cela répond aux objectifs de la loi du 4 mars 2002 et aux recommandations du Conseil de l'Europe, à savoir que "la participation des citoyens et des patients aux processus de décision concernant les soins de santé doit être considérée comme un droit fondamental de toute société démocratique » (Conseil de l'Europe, 2001). Les instances nationales (Haute Autorité de Santé, Direction Générale de l'offre de Soins, Direction Générale de la Santé) portent un intérêt particulier à cette thématique qui constitue une mesure phare du Programme National pour la Sécurité des Patients (PNSP). Au niveau international, l'OMS a lancé l'initiative "Patients for patients' safety" (PFPS), et vise à construire " $a$ world in which patients are treated as partners in efforts to prevent all avoidable harm in healthcare. PFPS calls for honesty, openness and transparency, and aims to make the reduction of healthcare errors a basic human right that preserves life around the world."

D'autre part, c'est en phase avec les souhaits des patients et des citoyens qui souhaitent être de plus en plus impliqués dans la gestion de leur propre santé (Coulter, \& Jenkinson, 2005 ; Coulter, \& Ellins, 2006).

6 Enfin, les patients eux-mêmes peuvent commettre des erreurs (Buetow, \& Elwyn, 2007) ou adopter des comportements à risques pouvant avoir une incidence négative sur leur propre sécurité, mais aussi sur la sécurité d'autres patients (Pernet, Mollo, \& Giraud, 2012; Pernet, 2013). En radiothérapie par exemple, le comportement à risque le plus prédominant est le fait qu'un patient prenne volontairement la place d'un autre, en déclinant une fausse identité, afin d'être traité plus rapidement. La conséquence directe peut être la réalisation du mauvais traitement, à la fois pour ce patient mais aussi pour d'autres patients. En étant mieux impliqués, les patients sont plus à même de comprendre les risques associés aux soins, ce qui permet d'éviter certains comportements à risque.

7 La présente recherche, conduite dans le domaine de la radiothérapie, considère le patient comme un acteur à part entière de la situation de soin, et non plus seulement comme un objet du soin. Elle s'intéresse aux facteurs qui permettent ou entravent le développement des capabilités des patients, c'est-à-dire aux actions que les patients sont réellement en mesure de réaliser pour éviter la survenue d'oublis ou erreurs, ou atténuer leurs effets.

Après avoir montré l'intérêt de considérer les patients comme des partenaires pour la sécurité des soins, nous définirons la "coopération» telle que nous l'entendons dans cette recherche et présenterons le modèle des capabilités que l'on a mobilisé pour comprendre la dynamique constructive de la coopération des patients. Nous présenterons ensuite la méthodologie déployée pour mettre en évidence les facteurs qui favorisent ou entravent la coopération des patients et donc la coopération soignant-soigné dans le contexte particulier qu'est la radiothérapie. Puis nous développerons ces facteurs. 


\section{Les patients : des partenaires potentiels pour la sécurité}

\subsection{Le soin : une activité coopérative}

9 Le soin est une forme particulière de situation de service où la production du service est vue comme une activité coopérative (Hoc, 1996 ; Guadagnoli, \& Ward, 1998). Pour Cerf, \& Falzon (2005), cette activité sous-entend la gestion concomitante d'actes techniques, d'initiatives éducatives et relationnelles et exige des compétences de diagnostic sur l'état de santé du patient ainsi que sur les compétences de coopération.

10 La coopération implique un partage du travail en fonction des ressources disponibles et des exigences immédiates du travail (de la Garza, \& Weill-Fassina, 2000). Elle n'implique pas obligatoirement l'exécution de tâches en commun, même si celles-ci convergent toujours vers un objectif unique. La coopération soignant-soigné est en grande partie intégrative (Schmidt, 1991) : elle vise à combiner des expertises, des savoir-faire ou des compétences différentes mais nécessaires pour réaliser la tâche. Du point de vue des patients, ils sont les seuls à pouvoir mesurer et partager l'impact de la maladie et des traitements sur leur qualité de vie. Ils disposent également d'une vision transverse du processus de soin (Vincent, \& Coulter, 2002 ; Koutantji, Davis, Vincent, \& Coulter, 2005 ; Davis, Jacklin, Sevdalis, \& Vincent, 2007 ; Hall, 2007 ; Unruh, \& Pratt, 2007) qui s'étend du temps d'hospitalisation (accueil, services de soins, etc.) aux soins à domicile et au suivi du traitement (médecine de ville, etc.). Une telle vision leur permet d'acquérir des connaissances et compétences sur leur propre maladie comme sur les effets indésirables et les comportements à adopter ou à éviter (Vincent, \& Davis, 2012), mais également sur les faiblesses et limites de la continuité des soins. Ils peuvent ainsi fournir des informations en temps réel qui permettent de détecter et récupérer des erreurs éventuelles qui, autrement, passeraient inaperçues (Unruh, \& Pratt, 2007; Vincent, \& Davis 2012). Par exemple, ils relayent des informations entre les médecins et les infirmières, rappellent les examens qui doivent être effectués, etc.

11 La coopération est fortement influencée par la nature de la relation qui s'instaure entre le soignant et le patient (Pernet, 2013). En effet, la dimension relationnelle de l'activité de soin, centrée sur le care, conditionne le développement d'une relation de confiance (Hesbeen, 1999). La relation de confiance qui sera instaurée permettra à son tour d'influencer la coopération, puisqu'elle crée les bases d'une collaboration constructive (Van Wijk, 2000). Ainsi, bien qu'elle relève du care et repose sur les compétences relationnelles des professionnels, la relation de confiance est également un déterminant du cure, puisqu'elle permet de coopérer en vue d'atteindre des objectifs de soins. Dit autrement, la relation de confiance impacte le pouvoir de coopération des patients. Nous y reviendrons plus tard.

\subsection{Coproduire la sécurité : les contributions des patients}

Davis, Sevdalis et Vincent (2011) définissent la participation des patients à la sécurité des soins comme les actions mises en œuvre par les patients pour réduire la probabilité d'erreurs médicales et/ou pour atténuer les effets des erreurs des soignants lorsqu'elles surviennent effectivement (2011). Du point de vue médico-légal, la participation des 
patients à la sécurité de leurs propres soins ne signifie pas qu'ils doivent en être responsables (WHO, 2013). Comme le souligne Lyons (2007), elle doit être perçue comme un moyen supplémentaire de renforcer la sécurité des soins, et non pas comme le moyen ultime.

Plusieurs travaux ont mis en avant la volonté des patients d'être impliqués dans la prévention des erreurs médicales (Weingart, \& al., 2005 ; Waterman, \& al., 2006 ; Pernet, 2010 ; Vincent, \& Davis, 2012; Martin, Nayne, \& Lipczak, 2013). Cette volonté est également présente chez les professionnels (Pernet, Mollo, \& Giraud, 2012; Martin, Nayne, \& Lipczak, 2013, Pernet, 2013). Selon l'étude menée par Weingart et al. (2004) auprès d'infirmières, ces dernières déclarent que l'implication des patients est un bon moyen de prévenir des erreurs médicales, notamment les erreurs médicamenteuses.

Par ailleurs, nos propres travaux (Pernet, 2013 ; Pernet, Mollo, Bibault, \& Giraud, 2016 ; Bibault, Pernet, Mollo, Gourdon, Martin, \& Giraud, 2016) ont montré que les patients sont capables de détecter et signaler des événements indésirables et que leur implication constitue une barrière de sécurité supplémentaire permettant de prévenir, détecter et récupérer certains oublis ou erreurs des soignants. Cependant, cette implication ne peut être efficace que si elle repose sur la coopération soignant/soigné, elle-même déterminée par la construction d'une relation de confiance. C'est pourquoi nous parlerons de coopération plutôt que de participation des patients à la sécurité des soins, dans la mesure où les contributions potentielles des patients prennent place dans un rapport de place coopératif qui est déterminé par :

- Un engagement réciproque des partenaires dans la coproduction d'un soin sûr et efficace. Cet engagement est conditionné notamment par la volonté d'implication de chacun des partenaires (volonté du patient d'être impliqué dans le soin, et volonté du professionnel d'impliquer le patient) et une conscience mutuelle du rôle de chacun (incluant la prise en compte des capacités du patient à pouvoir coopérer: langue parlée, capacité de compréhension...).

- Le contexte de travail dans lequel se construit la relation.

15 En effet, au-delà des questions de volonté et de capacité, l'implication des patients est fortement dépendante de la possibilité réelle qui leur est donnée de coopérer à la production d'un soin sûr et efficace.

\section{De la capacité à coopérer à la possibilité de coopérer : le modèle des capabilités}

L'approche des capabilités développée par Sen $(1999,2009)$ offre une lecture intéressante des contributions possibles des patients à la sécurité des soins.

Cette approche est illustrée dans la figure 1. 
Figure 1. Illustration de l'approche des capabilités.

Figure 1. Illustration of the capability approach

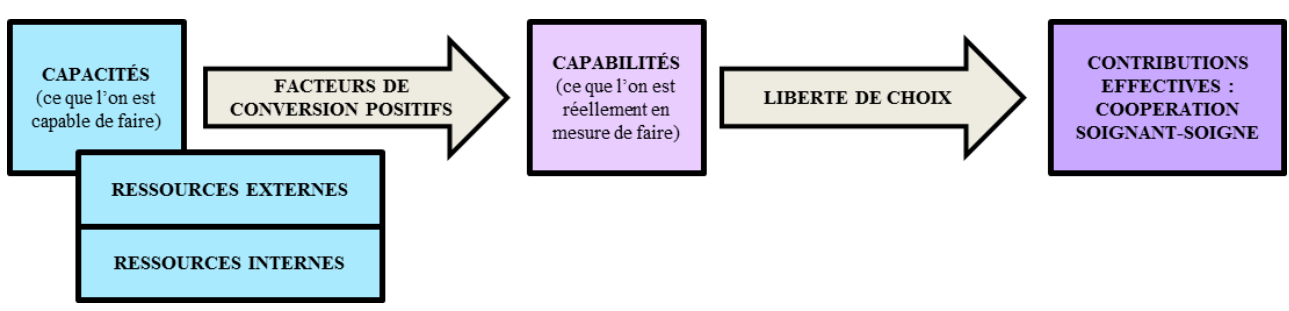

18 Les capacités renvoient à ce qu'une personne, le patient en l'occurrence, est capable de faire (Falzon, \& Mollo, 2009; Six-Touchard, \& Falzon, 2013). Elles dépendent des ressources dont le patient dispose, qu'elles soient internes (ex: capacités cognitives et acceptation de la maladie) ou externes (ex : l'environnement et la durée du traitement). Les capabilités renvoient quant à elles, à ce que le patient est réellement en mesure de faire (Falzon, \& Mollo, 2009 ; Six-Touchard, \& Falzon, 2013). Les contributions effectives, quant à elles, renvoient à ce qui est effectivement réalisé.

Les notions de capacité et de capabilité sont implicitement liées mais une capacité ne suffit pas à permettre une capabilité : des facteurs de conversion sont nécessaires à cet effet. Ces derniers renvoient à «l'ensemble des facteurs qui facilitent ou entravent la capacité d'un individu à faire usage des ressources à sa disposition pour les convertir en réalisation concrètes » (Fernagu-Oudet, 2012). Nous parlerons de facteurs de conversion positifs lorsqu'ils permettent la transformation d'une capacité en capabilité. À l'inverse, nous parlerons de facteurs de conversion négatifs lorsqu'ils entravent ou empêchent la transformation d'une capacité en capabilité.

La capabilité traduit le pouvoir effectif de choisir (Arnoud, \& Falzon, 2013) mais est aussi le fruit d'une organisation de travail. En effet, la situation de travail doit offrir une liberté de choix aux patients, qu'ils coopèrent ou non. Ainsi la capabilité repose sur le concept même de pouvoir d'agir (Mollo, \& Falzon, 2009) qui se définit comme «la possibilité effective de mettre en œuvre une capacité donnée ». Selon Doumont et Aujoulat (2002), le pouvoir d'agir désigne « la capacité d'un individu à prendre des décisions et à exercer un contrôle sur sa vie personnelle ». Développer le pouvoir d'agir des patients consiste à renforcer les capacités des patients de façon à leur permettre de participer activement à la gestion de leurs soins et de contribuer plus largement à la gestion du système de santé (Lau, 2002).

\section{Contexte de l'étude : la sécurité en radiothérapie}

21 La radiothérapie est un traitement locorégional des cancers impliquée dans $60 \%$ des traitements des patients atteints de cancer. En 2009 et 2010, environ 180000 patients ont été pris en charge par radiothérapie en France (ASN, 2011a et b).

La production d'un soin sûr et efficace dans cette spécialité médicale repose sur 5 critères de sécurité : administrer le «Bon traitement », la «Bonne dose », au «Bon endroit», au «Bon moment » au «Bon patient ». Amener les patients à coopérer lors de leurs séances de traitement peut permettre de prévenir des événements mettant en danger ces critères. 


\subsection{Les principales étapes du parcours des patients en radiothérapie}

Le parcours des patients en radiothérapie comprend 6 grandes étapes :

- La consultation médicale initiale : le radiothérapeute explique au patient le traitement, les effets secondaires possibles et les recommandations à suivre ;

- Le temps d'accompagnement soignant : réalisée par un manipulateur, cette phase consiste à informer le patient sur le déroulement des séances de traitement, les effets secondaires et les recommandations à suivre ;

- Le scanner de dosimétrie : un soignant détermine la zone à traiter et les organes à protéger en vue de définir la dose ;

- La mise en place : une séance «à blanc » qui permet une vérification du positionnement du patient et de la position des faisceaux d'irradiation;

- Le traitement : Le traitement en radiothérapie est un traitement long qui s'étale sur 5 à 7 semaines, à raison de 5 séances par semaine en moyenne. Les séances sont associées à des consultations médicales hebdomadaires avec le radiothérapeute;

- La consultation médicale de fin de traitement : le radiothérapeute met en place un suivi de la prise en charge.

Les séances de radiothérapie durent de 10 à 20 minutes en fonction de la localisation à traiter et de la complexité du traitement. Ce temps comprend le déshabillage en cabine, le positionnement sur la table de traitement, la vérification du positionnement par le manipulateur et la réalisation du traitement proprement dit (3 à 5 minutes). Durant son traitement, les patients ont également des consultations hebdomadaires avec leur radiothérapeute référent.

\subsection{Les contributions effectives des patients à la sécurité des soins}

Une première série de travaux a mis en évidence les contributions effectives des patients lors des séances de traitement (Mollo, Pernet, Moutel, Duchange, \& Giraud, 2011 ; Pernet, Mollo, \& Giraud, 2012; Pernet, 2013). Elles peuvent se traduire par des actions préventives ou correctives (Pernet, 2013).

Les actions préventives consistent à prévenir l'apparition d'oubli et ou erreur, ou à favoriser la réalisation d'un traitement dans les meilleures conditions possibles. Par exemple :

- La vérification de son identité sur l'écran situé en salle de traitement,

- La vérification par le patient du nombre de faisceaux de traitement et du temps d'irradiation pour chaque faisceau de traitement,

- La reproduction du positionnement attendu avant la séance et le respect de l'immobilité durant la séance,

- Le respect de la temporalité des séances de traitement sans interrompre son traitement.

Par exemple, la vérification de son identité sur l'écran permet au patient de s'assurer qu'il reçoit bien le traitement qui lui est assigné. Elle garantit ainsi que le «Bon patient » reçoit le «Bon traitement ». De même, la reproduction du positionnement attendu et le respect de l'immobilité permettent au patient de garantir le critère de sécurité «Bon endroit ». 

aura tendance à s'impliquer dans la sécurité de son traitement. À l'inverse, un patient qui n'a pas accepté sa maladie aura tendance à subir son traitement : il restera en retrait lors de ses séances. Il sera présent physiquement mais absent mentalement. De plus, les patients curieux, qui cherchent à comprendre, ont tendance à être plus impliqués dans leur traitement. D'autre part, la coopération requiert des capacités cognitives comme la compréhension du traitement par le patient. Elle requiert également des capacités physiques. Par exemple, la vérification d'identité sur l'écran en salle de traitement demande que le patient soit voyant. Enfin, l'absence de douleur chronique est plus favorable à l'implication des patients alors que les patients algiques sont plus centrés sur leur douleur que sur la réalisation du traitement. C'est le cas des patients traités en palliatifs.

Les actions correctives, quant à elles, consistent à signaler tout oubli et/ou erreur concernant les contentions utilisées (aides au positionnement du patient), le positionnement attendu, l'identité du patient, la zone à irradier.

les patients contribuent à la sécurité et l'efficacité du traitement. La section suivante présente les ressources qui permettent la réalisation effective de ces contributions.

\subsection{Les ressources internes et externes}

Deux catégories de ressources peuvent expliquer que les patients sont capables - ou non de coopérer dans la sécurité et l'efficacité de leur traitement: les ressources internes et les ressources externes (Pernet, 2013). Ces ressources ne sont pas figées; elles sont variables d'une situation à l'autre et sont susceptibles d'évoluer tout au long de la prise en charge. Par exemple, un patient peut très bien ne pas avoir accepté sa maladie en début de traitement et finir par l'accepter au cours de son traitement.

maladie sera moins stressé, moins angoissé, et aura tendance à s'impliquer dans la sécurité de son traitement. À l'inverse, un patient qui n'a pas accepté sa maladie aura tendance à subir son traitement : il restera en retrait lors de ses séances. Il sera présent physiquement mais absent mentalement.

- La curiosité et la volonté de s'en sortir ; les patients curieux, qui cherchent à comprendre, ont tendance à être plus impliqués dans leur traitement.

- Les capacités cognitives, physiques et l'absence de douleur chronique.

Les ressources externes, quant à elles, renvoient à l'environnement et à la temporalité de la radiothérapie.

L'environnement correspond à la conception de la salle et aux dispositifs techniques. Il offre un horizon d'observation qui permet au patient de s'approprier les informations qu'il contient afin :

1. De le renseigner sur l'activité du manipulateur (voir ce que fait le manipulateur),

2. D'avoir une visibilité de son nom sur l'écran,

3. D'avoir une visibilité des mouvements de l'accélérateur,

4. D'avoir la possibilité d'entendre les phases d'irradiation. 
poralité de la radiothérapie est variable, mais implique une répétition des séances de traitement (tous les jours pendant plusieurs semaines) qui permet au patient d'apprendre de la situation et d'acquérir des connaissances sur son traitement (par exemple sur les contentions utilisées, le positionnement sur la table de traitement, le nombre de faisceaux de traitement, les mouvements de l'accélérateur). Ces connaissances permettent au patient de détecter d'éventuels oublis et/ou erreurs, voire des variations de manière de faire.

L'environnement et la temporalité de la radiothérapie permettent un apprentissage de la situation, offrant une opportunité créatrice au patient d'évaluer la situation en observant ce qui se passe.

Toutes ces ressources sont des déterminants nécessaires à la coopération des patients dans la sécurité et l'efficacité de leur traitement. Cependant, les ressources internes ne sont pas figées ; elles peuvent évoluer tout au long de la prise en charge thérapeutique, de même que de nouvelles ressources pourront être créées comme la volonté de coopération, la relation coopérative ou le rapport de place coopératif qui offre une liberté, un droit d'expression (Pernet, 2013). L'enjeu est de donner la possibilité aux patients de pouvoir coopérer s'ils le désirent, quel que soit leur profil. Le niveau de coopération du patient peut donc évoluer tout au long de sa prise en charge.

La figure 2 reprend les éléments mis en évidence jusqu'ici.

Figure 2. L'approche des capabilités : ressources, ressources créées, actions.

Figure 2. The capability approach : resources, created resources, associated actions

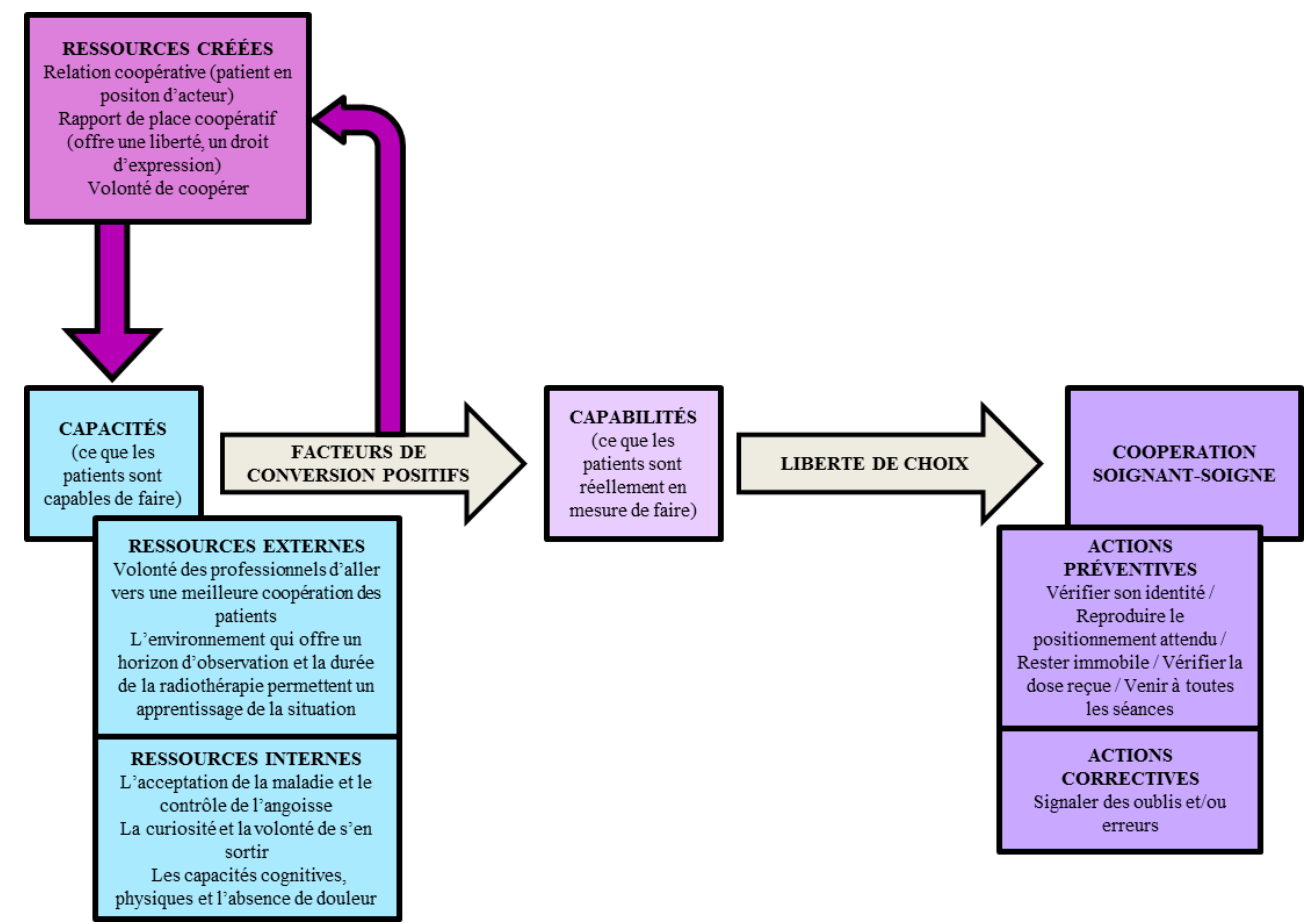




\section{Objectif et méthodologie}

\subsection{Objectif} permettent de coopérer. Ces résultats, synthétisés ci-dessus, sont détaillés dans Pernet
(2013).

42 Pour que les patients coopèrent à la sécurité et l'efficacité des soins, il est essentiel qu'ils disposent de ressources et de pouvoir d'agir. Cependant, il apparaît également primordial de donner du pouvoir d'agir aux professionnels pour qu'ils puissent amener les patients à contribuer à la sécurité. Ces éléments expliquent l'intérêt de mobiliser l'approche des capabilités puisqu'elle repose sur le concept même de pouvoir d'agir. De plus, cette approche nous a permis de mettre en évidence les ressources internes et externes aux patients, ainsi que les facteurs de conversion nécessaires à la coopération soignant-soigné et d'en comprendre sa dynamique constructive.

43 L'objectif de ce papier est de comprendre la dynamique de développement des capabilités des patients. Il s'agit d'analyser les facteurs de conversion qui favorisent ou entravent le développement de ces capabilités, c'est-à-dire les facteurs qui rendent possible ou empêchent la coopération des patients dans la sécurité et l'efficacité de leur traitement.

\subsection{Méthodologie}

44 Pour ce faire, plusieurs méthodes ont été mises en œuvre: des observations systématiques de séances de traitement, des autos et allo-confrontations individuelles (Mollo, \& Falzon, 2004) réalisées auprès de manipulateurs et des entretiens menés auprès de patients. La combinaison de ces méthodes a permis d'analyser à la fois l'activité des professionnels, l'activité des patients et les interactions professionnels-patients.

\subsubsection{Des observations systématiques de séances de traitement}

16 journées de travail de 16 manipulateurs ont été observées, sur un poste complet (8 journées dans chacune des deux structures). Ces observations se sont focalisées sur l'étape du traitement pour deux raisons majeures. D'une part, les événements indésirables (EI) en radiothérapie surviennent fréquemment lors de cette phase. En effet, sur les 242 EI déclarés à l'ASN en 2011, plus de la moitié renvoient à une erreur de positionnement (55\%). $21 \%$ de ces EI renvoient à une erreur d'identification du patient, $12 \%$ à une erreur de dose et $12 \%$ à une erreur de géométrie (ASN, 2013). D'autre part, c'est à cette phase que la coopération soignant-soigné est déterminante pour garantir la sécurité et l'efficacité des soins. En effet, le patient et le manipulateur doivent obtenir un positionnement qui soit le plus précis possible, et qui garantisse l'immobilité du patient durant la séance. 
Ces observations avaient pour objectifs :

- D'analyser l'activité du manipulateur et du patient, et plus précisément les modalités de coopération mises en œuvre.

- De mettre en évidence les différents aspects du contexte qui peuvent modifier la coopération et avoir des répercussions sur la relation manipulateur-patient.

Au total, 113 heures d'observation ont été effectuées, dont 46 heures au CHU et 67 heures au CLCC. Concernant plus spécifiquement le positionnement des patients, 126 séances ont été observées au CHU et 110 au CLCC.

Trois grandes catégories d'observables ont été recueillies et analysées.

Tout d'abord, les interactions manipulateur-patient ont été enregistrées et analysées via une analyse thématique. 3 grandes classes de variables ont été étudiées :

- Le locuteur et l'interlocuteur : identification de la personne initiatrice de l'interaction et du destinataire de ce message.

- La nature des interactions : il pouvait s'agir d'une requête ou d'un apport d'informations.

- Le contenu des interactions selon qu'elles renvoient :

- À la coordination et à l'accomplissement des actions ;

- Au rappel des consultations à venir (avec le médecin, le psychologue, le psychiatre, le diététicien);

- Aux éléments visant à amener le patient à coopérer ;

- Aux informations et explications sur le déroulement du traitement, les caractéristiques du traitement qui permettent à la fois aux patients d'acquérir des connaissances sur leur traitement et de se rassurer.

En parallèle du codage des communications, les actions des patients (P) et des manipulateurs (M) ont été codées en respectant leur chronologie, à savoir respectivement : préparation de la table de traitement; positionnement; délivrance du traitement; fin de traitement.

51 Pour finir, une classification des situations de travail a été établie en fonction des horaires de prise en charge des patients afin de comprendre l'impact du contexte de travail sur le développement des capabilités des patients. Deux grands types de situations ont été distingués :

- Les situations standards : les séances se déroulent à l'heure prévue (patient pris à l'heure et patient pris avec un retard inférieur à 15 minutes) ou en avance par rapport au planning prescrit de la journée.

- Les situations dégradées: les séances ont pris du retard (retard supérieur ou égal à 15 minutes).

52 On a considéré que les manipulateurs se trouvaient en situation dégradée lorsque l'horaire de prise en charge du patient est supérieur ou égal à 15 minutes par rapport à l'horaire prescrit. En effet, c'est le retard à partir duquel on peut observer un changement de l'activité des manipulateurs. De plus, d'après les manipulateurs et les patients de l'étude, un retard inférieur à 15 minutes est acceptable pour les deux parties.

\subsubsection{Des auto et allo-confrontation individuelles auprès de manipulateurs}

53 L'analyse - qualitative et quantitative - des observations systématiques a été complétée par des entretiens d'auto et allo confrontations individuelles (Mollo, \& Falzon, 2004) auprès de 20 manipulateurs (10 dans chacun des établissements de soins). L'intérêt était 
d'identifier, comprendre et approfondir les facteurs de conversion qui favorisent ou entravent le développement des ressources et des capabilités des patients. Plus précisément, il s'agissait de comprendre :

- En quoi la situation discutée est propice ou non à la coopération des patients dans la sécurité et l'efficacité des soins,

- En quoi la relation de confiance permet de développer une relation coopérative et de révéler ou renforcer le potentiel d'action des patients,

- Les déterminants de la construction d'une relation de confiance ainsi que les moyens mis en place pour pouvoir construire cette relation,

- L'impact des pressions temporelles sur l'activité des manipulateurs.

L'auto-confrontation individuelle consiste à demander à un manipulateur de commenter sa propre activité à partir de traces de celle-ci. Elle lui permet d'expliciter les raisons des actions. L'allo-confrontation individuelle consiste à demander à un manipulateur de commenter l'activité d'un collègue (un autre manipulateur de leur établissement ou de l'autre établissement participant à l'étude), à partir de traces de cette activité. Ces dernières sont des supports papier illustrant des situations observées. Chaque situation reprenait le contexte (date, heure, etc.), les actions (positionnement, traitement, etc.) et les échanges manipulateurs-patients (cf. annexe 1). Auto et allo-confrontations ont été retranscrites intégralement et traitées à partir d'une analyse thématique.

\subsubsection{Des entretiens réalisés auprès de patients}

Pour analyser les facteurs de conversion expliquant la coopération des patients, deux entretiens ont été réalisées auprès de 20 patients : le premier en début de traitement, le second en fin de traitement, afin de prendre en compte l'évolution des relations et des connaissances des patients au cours du temps.

Afin d'homogénéiser le recueil et l'analyse des informations, deux pathologies cancéreuses ont été choisies en raison de leur fréquence et du caractère emblématique de la localisation : le cancer du sein chez la femme et le cancer de la prostate chez l'homme. Dix de ces 20 patients ( 5 femmes et 5 hommes) sont issus du CHU et les 10 autres du CLCC. La répartition hommes/femmes est homogène dans les deux établissements soit 5 femmes et 5 hommes dans le CHU et 5 femmes et 5 hommes dans le CLCC.

Chaque entretien a été retranscrit intégralement et a fait l'objet d'une évaluation qualitative par analyse thématique. Une série de thèmes relatifs aux questions posées a été préalablement élaborée (volonté de coopération, confiance, etc.), puis adaptée aux réponses obtenues.

\section{Résultats}

Les résultats sont présentés en deux parties. La première détaille les facteurs de conversion qui soutiennent le développement des capabilités des patients. La seconde montre le rôle du contexte de travail sur la coopération des patients. 


\subsection{Les facteurs de conversion soutenant le développement des capabilités des patients}

Il ne suffit pas que le patient dispose de ressources externes et internes pour être amené à coopérer dans la sécurité et l'efficacité de son traitement : il faut également des facteurs de conversion qui permettent de transformer les capacités des patients en capabilités. Trois grandes classes de facteurs positifs ont pu être distinguées :

- La connaissance des risques associés aux soins (facteurs générés par les patients),

- La relation de confiance (facteur généré par le binôme manipulateur-patient),

- L'encouragement et le renforcement positif (facteurs générés par les soignants).

\subsubsection{La connaissance des risques associés aux soins et la motivation personnelle}

La connaissance des risques associés aux soins est un premier facteur positif puisqu'elle conduit les patients à mesurer l'importance de coopérer.

Tous les manipulateurs s'accordent à dire qu'il y a une certaine conscience des risques chez les patients, plus au moins importante d'un patient à l'autre, qui explique le comportement coopératif du patient. Selon eux, les patients qui coopèrent dans la sécurité et l'efficacité de leur traitement ont une connaissance préalable des risques associés aux soins, notamment au travers des médias (par exemple : erreurs d'irradiation, erreurs de site opératoire). Cette conscience des risques conduit les patients à mesurer l'importance de s'impliquer et de coopérer afin de prévenir les risques. Certains patients expliquent que cette vigilance n'est pas associée à un sentiment d'incompétence des professionnels mais à une organisation de travail et des conditions de travail mises à mal dans les établissements de soins qui engendrent des risques associés aux soins.

" Tout le monde est au courant des erreurs qui surviennent dans les structures de soins. On en parle à la télévision. Pour mon cas, je pense que ça m'amène à être attentif lors de mon traitement ». Paroles d'un patient

« De nombreux drames sont survenus dans les hôpitaux. La presse et les chaînes d'information en rapportent constamment. Ça nous amène à rester vigilants. Cependant, il est important de dire que dans la majorité des cas ce n'est pas dû à un manque de compétences des professionnels mais à des questions d'organisation et de conditions de travail qui sont parfois déplorables ». Paroles d'une patiente.

Cette connaissance des risques renvoie :

- À la conscience de la survenue d'erreurs dans les établissements de soins,

- Au fait qu'un mauvais positionnement et le non-respect de l'immobilité auront des effets négatifs sur la sécurité et l'efficacité du traitement,

- Au fait qu'une interruption de traitement impactera l'efficacité du traitement.

La motivation personnelle est un deuxième facteur de conversion positif qui amène les patients à coopérer afin de garantir la sécurité et l'efficacité de leur traitement et de prendre un certain contrôle sur leur maladie. La coopération apparaît donc comme un moyen de contrôler la maladie.

«Vous savez pour moi outre le fait que je dois être impliquée pour la sécurité de mon traitement et sa réussite, mon implication me permet un contrôle sur la maladie en quelque sorte. La maladie a pris le contrôle de mon corps à un moment donné et bien maintenant c'est moi qui prend le contrôle en me faisant traiter et en étant impliquée ». Paroles d'une patiente. 
« Les patients valides, qui ont envie de s'en sortir sont impliqués, et nous on va dans ce sens-là. Ça les stimule. Je pense que pour eux le fait de s'impliquer c'est une sorte de maîtrise sur le cancer ». Paroles d'une manipulatrice.

Cette connaissance des risques et la motivation personnelle expliquent que certains patients font le choix de coopérer et qu'ils mettent en œuvre des actions préventives afin de coproduire un soin sûr et efficace.

\subsubsection{La relation de confiance}

La construction d'une relation de confiance est un facteur de conversion positif majeur puisqu'elle permet de développer une relation coopérative.

6 Pour que le patient puisse avoir confiance envers le manipulateur, il doit avant tout pouvoir le juger compétent dans sa fonction. Tous les patients expliquent observer les manipulateurs dans la réalisation de leurs différentes tâches. La précision qui est manifeste dans leur activité explique que les patients les perçoivent comme des professionnels compétents. Certains patients attendent également du professionnel qu'il soit honnête et les informe sur l'ensemble des effets secondaires connus.

Outre la compétence et l'honnêteté, les qualités relationnelles jouent également un rôle essentiel dans la construction d'une relation de confiance. Elles renvoient au travail du care qui permet aux patients de se sentir reconnus, écoutés et respectés.

D'après les manipulateurs, chaque relation est différente dans la mesure où elle dépend des caractéristiques du patient, de sa personnalité, de son ouverture d'esprit, de son état psychologique et physique et de ses attentes. Dans ce contexte, l'objectif des manipulateurs est de connaître le patient, de comprendre ses attentes et de construire une attitude thérapeutique adaptée. Cette capacité d'adaptation s'acquiert par l'expérience et s'enrichit constamment par les situations rencontrées.

L'instauration d'une relation de confiance est avant tout déterminée par la capacité des manipulateurs à répondre aux attentes spécifiques des patients. Mais la confiance accordée aux manipulateurs doit être "raisonnée » et non aveugle. Dans le cas d'une confiance aveugle, le patient est passif car il assigne la place d'acteur aux seuls manipulateurs ; une relation coopérative sera alors impossible. La confiance « raisonnée » implique une vigilance constante du patient et rend possible le développement d'une relation coopérative (voir figure $n^{\circ} 3$ ). 
Figure 3. Les caractéristiques de la confiance aveugle et de la confiance raisonnée. Figure 3. The characteristics of blind trust and of reasoned trust

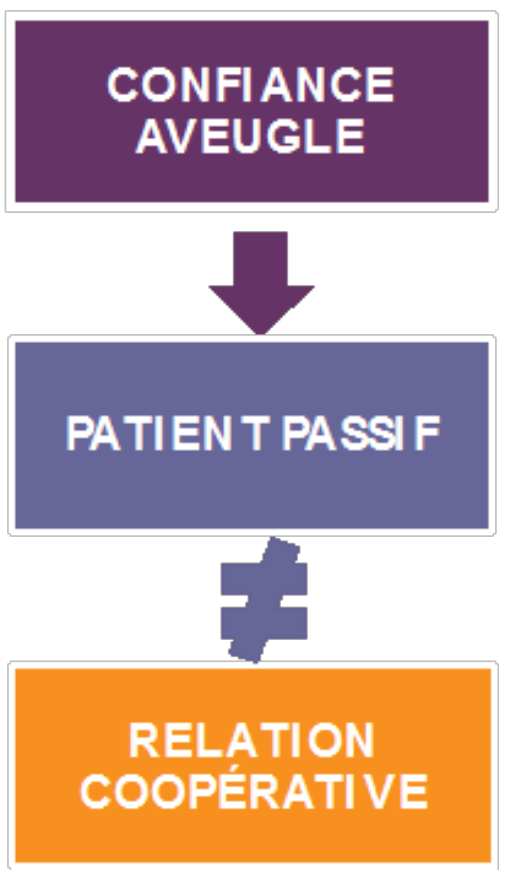

\section{CONFIANCE RAISONNÉE}

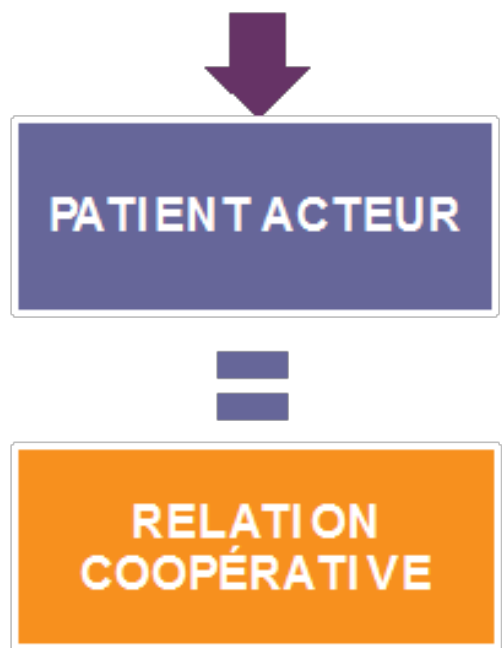

Cette confiance « raisonnée » est rendue possible par la connaissance que les patients ont des risques associés aux soins. Les patients expliquent que des erreurs peuvent survenir, que l'erreur est humaine et qu'elle ne remet pas en cause la compétence du manipulateur. Ils ont tendance à expliquer la survenue des erreurs par l'organisation du travail et plus particulièrement par les contraintes temporelles.

«Si je remarque quelque chose d'inhabituel, je le signalerai. Ça peut être une erreur. L'erreur est humaine. Il est important de signaler. Il faut faire confiance car ils sont très compétents mais il faut avoir un œil ouvert. Ce n'est pas de la méfiance mais malheureusement c'est comme dans les avions ce n'est pas parce que vous allez avoir le meilleur pilote du monde que les erreurs ne peuvent exister ». Paroles d'un patient.

«Si je constate quelque chose d'inhabituel, je le signalerai évidemment. J'imagine que ça peut être une erreur. Les erreurs peuvent arriver même si vous savez qu'ils sont compétents. J'ai bien conscience qu'ils ont plein de patients à traiter dans un temps restreint donc une erreur peut arriver ». Paroles d'un patient.

Les données recueillies ont permis de mettre en évidence les intérêts de la relation de confiance dans la coopération soignant-soigné.

Premièrement, la relation de confiance offre une liberté d'expression au patient. Cette dernière est indispensable pour oser poser des questions ou signaler quelque chose d'inhabituel. En effet, si certains patients déclarent qu'ils signaleraient tout événement inhabituel même s'il n'y pas de relation de confiance instaurée, d'autres au contraire n'osent pas intervenir.

Deuxièmement, la relation de confiance traduit une personnalisation du soin qui apparaitt comme une forme de reconnaissance du patient mais également comme une forme d'identitovigilance qui permet de s'assurer qu'il s'agit bien du «bon patient » et que celui-ci reçoit bien le «bon traitement». Plusieurs des manipulateurs soulignent qu'appeler le patient par son nom de famille est non seulement rassurant mais aussi très 
sécurisant. Pour les patients, cette personnalisation du soin évite les erreurs d'identité et permet le contrôle du traitement reçu.

Troisièmement, la relation de confiance est un moyen de garantir la qualité du positionnement (précision et maintien de l'immobilité). D'après les manipulateurs, la relation qui se construit avec les patients influence le stress et l'angoisse de ces derniers, et ce faisant leur rigidité corporelle. Ainsi, plus le patient est détendu, plus il est facile d'aboutir à un positionnement précis qui soit confortable pour le patient et maintenable pendant la délivrance du traitement. Face à un patient stressé, les manipulateurs arrivent tout de même à reproduire le positionnement attendu, même si cela prend plus de temps, mais il est difficile de garantir l'immobilité du patient durant l'irradiation. Le patient peut se contracter encore plus lorsqu'il est seul dans la salle de traitement. Le stress ou l'angoisse $\mathrm{du}$ patient sont donc des facteurs qui interfèrent sur la qualité du positionnement, et ce faisant sur la sécurité du traitement. La relation de confiance est un moyen de pouvoir les dépasser.

Enfin, tous les patients interviewés déclarent que la relation instaurée avec les manipulateurs les motive à venir à leurs séances de traitement.

La construction d'une relation de confiance permet donc d'instaurer un climat d'échange qui rend le patient acteur et soutient le développement d'une relation coopérative manipulateur-patient. Le care apparait comme une condition du cure qui permet la coopération des patients dans la coproduction d'un soin sûr et efficace. En retour, la coopération renforce la confiance.

\subsubsection{L'encouragement et le renforcement positif}

Le premier facteur de conversion généré par les soignants est l'encouragement à coopérer, qui peut prendre trois formes. Tout d'abord, l'encouragement peut porter sur la compliance du patient, c'est-à-dire le respect par ce dernier des recommandations qui lui sont adressées: mémoriser son positionnement, rester immobile durant l'irradiation, venir à toutes les séances de traitement. L'encouragement peut également concerner la vigilance du patient : vérifier son identité sur l'écran, repérer les choses qui paraissent inhabituelles. Enfin, l'encouragement peut concerner la liberté d'expression du patient, en signalant les choses qui lui paraissent inhabituelles.

L'encouragement marque donc une possibilité réelle de coopérer que les patients sont libres d'accepter ou pas.

Le tableau 1 ci-dessous illustre ces trois formes d'encouragement. 
Tableau 1. Formes d'encouragement.

Tableau 1. Forms of encouragement

\begin{tabular}{|c|c|c|}
\hline \multirow{4}{*}{$\begin{array}{l}\text { Encouragement } \\
\text { fondé sur la } \\
\text { compliance }\end{array}$} & \multirow{2}{*}{$\begin{array}{l}\text { À mémoriser et à } \\
\text { reproduire le } \\
\text { positionnement } \\
\text { attendu }\end{array}$} & $\begin{array}{l}\text { "Au scanner de dosimétrie, le manipulateur m'a bien expliqué que cette position } \\
\text { dans laquelle j'étais, serait exactement la même que lors des séances de traitement. } \\
\text { Du coup, j'ai été attentive à retenir la façon dont j'étais positionnée pour pouvoir } \\
\text { me mettre au mieux lors des séances ». Paroles d'une patiente }\end{array}$ \\
\hline & & $\begin{array}{l}\text { "Par exemple pour les cale-pieds, on leur demande qu'ils soient bien au bout, de } \\
\text { veiller à le faire tous les jours, mais c'est vrai qu'aussi ils le font d'eux-mêmes. Ils } \\
\text { savent très bien que s'ils sont bien positionnés, le traitement sera mieux fait 》. } \\
\text { Paroles d'une manipulatrice }\end{array}$ \\
\hline & À rester immobile & $\begin{array}{l}\text { "C'est vrai qu'on leur dit tout le temps, une fois le patient positionné : "ne bougez } \\
\text { plus on y va". On le dit même à des patients en fin de traitement qui ont bien } \\
\text { compris qu'il ne fallait pas bouger. Mais bon c'est quelque chose de tellement } \\
\text { important, que ça en est devenu un réflexe dans notre pratique ». Paroles d'une } \\
\text { manipulatrice }\end{array}$ \\
\hline & $\begin{array}{l}\text { À respecter la } \\
\text { temporalité }\end{array}$ & $\begin{array}{l}\text { "Mon radiothérapeute m'a informée lors de la consultation médicale, avant le } \\
\text { commencement des séances de traitement, de l'importance de venir à toutes les } \\
\text { séances pour garantir l'efficacité de mon traitement ». Paroles d'une patiente }\end{array}$ \\
\hline \multirow{2}{*}{$\begin{array}{l}\text { Encouragement } \\
\text { fondé sur la } \\
\text { vigilance }\end{array}$} & \multirow{2}{*}{$\begin{array}{l}\text { À vérifier son } \\
\text { identité }\end{array}$} & $\begin{array}{l}\text { "Avant chaque début de séance de traitement, je regarde bien que mon nom soit } \\
\text { indiqué pour éviter toute éventuelle erreur d'identité. On me l'a demandé lors de } \\
\text { ma première séance. Je trouve ça bien. Je sais que les manipulateurs vérifient aussi } \\
\text { mais vaut mieux deux vérifications qu'une seule comme on dit ». Paroles d'une } \\
\text { patiente }\end{array}$ \\
\hline & & $\begin{array}{l}\text { "Les patients regardent que ça soit bien leur nom marqué sur l'écran et c'est vrai } \\
\text { qu'on leur dit. On aime bien leur dire et les faire vérifier parce que l'on peut } \\
\text { oublier. Le fait de regarder son mon, ce n'est pas grand-chose mais tu as déjà } \\
\text { l'impression de dominer ton traitement et c'est super important. Pour moi ce cốé } \\
\text { acteur, c'est quelque chose que l'on doit développer ». Paroles d'un manipulateur }\end{array}$ \\
\hline $\begin{array}{l}\text { Encouragement } \\
\text { fondé sur la } \\
\text { liberté } \\
\text { d'expression }\end{array}$ & $\begin{array}{l}\text { À signaler les } \\
\text { choses qui } \\
\text { paraissent } \\
\text { inhabituelles }\end{array}$ & $\begin{array}{l}\text { "J'ai une espèce de mousse en silicone, qu'on me met au niveau du coccyx pour } \\
\text { éviter d'avoir mal sur la table qui est assez dure. Les manipulateurs m'ont dit en } \\
\text { début de traitement de ne pas hésiter à leur rappeler de la mettre. Je sais que c'est } \\
\text { important car ça me permet de ne pas avoir mal et d'être détendue. En l'absence de } \\
\text { cette mousse, je pourrais avoir mal, je serais donc contractée, ce qui pourrait } \\
\text { fausser mon positionnement ». Paroles d'une patiente }\end{array}$ \\
\hline
\end{tabular}

Le deuxième facteur de conversion généré par les soignants est le renforcement positif. Il fait suite à une action réalisée par le patient en termes de sécurité des soins et consiste à féliciter les patients de cette action. Pour exemple, un manipulateur qui dit au patient: "C'est bien de participer à la vérification de son identité ». À travers le renforcement positif, le manipulateur cherche à renforcer le comportement actif du patient déjà existant, ce qui augmente la probabilité que le patient puisse coopérer à d'autres niveaux.

81 L'encouragement et le renforcement positif traduisent d'une certaine éducation des patients dans la coopération d'un soin sûr et efficace.

82 La figure 4, ci-dessous, reprend les facteurs de conversion positifs analysés, qui offrent aux patients la possibilité de coopérer. 
Figure 4. L'approche des capabilités : ressources, ressources crées, facteurs de conversion et actions associées.

Figure 4. The capability approach: resources, created resources, conversion factors and associated actions

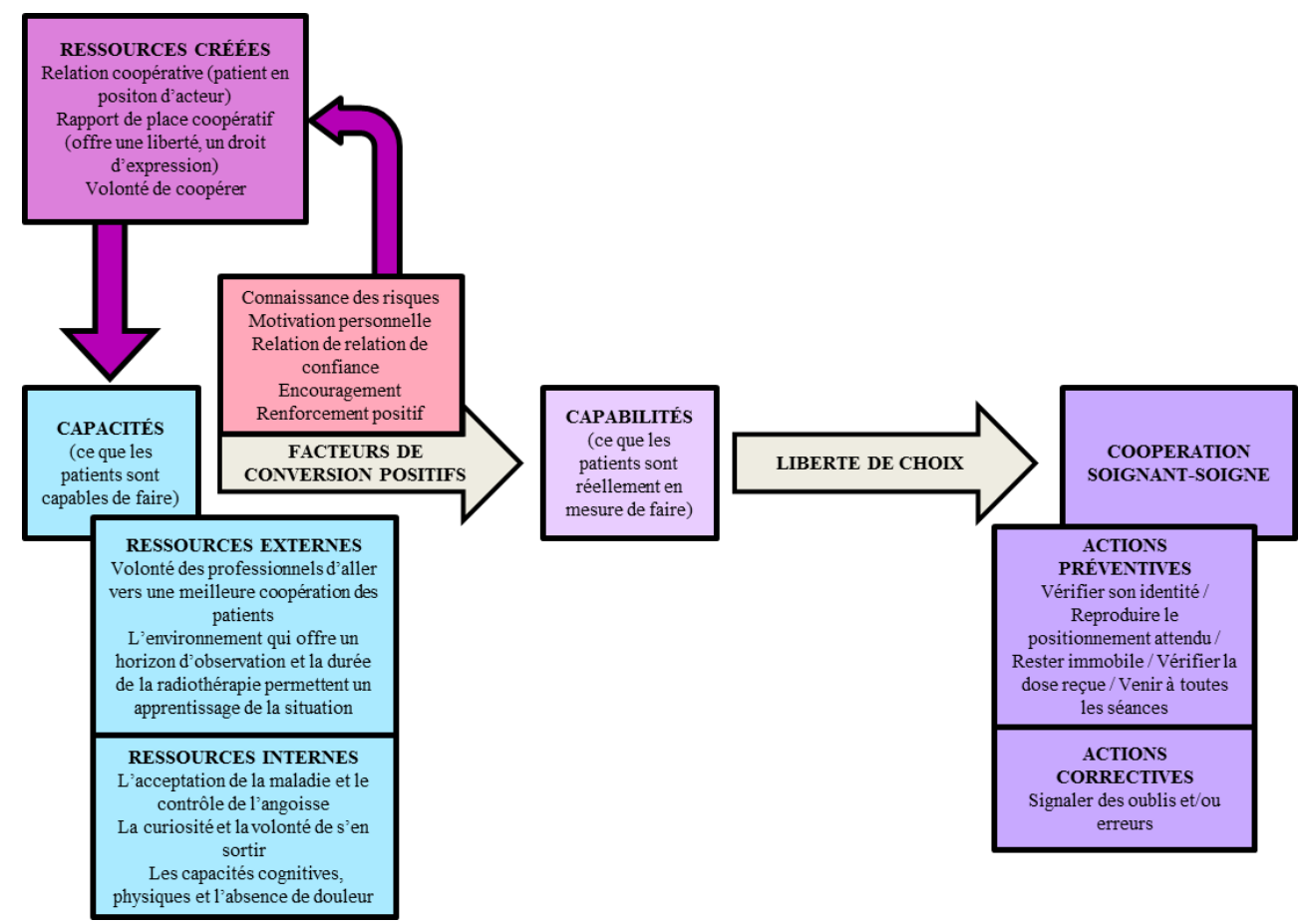

\subsection{L'impact du contexte de travail sur le développement des capabilités}

83 L'analyse de l'activité a mis en évidence l'importance du contexte dans le développement des capabilités et ainsi dans le développement d'une relation coopérative. Les situations dégradées (présentant un retard supérieur à 15 minutes) semblent notamment empêcher et/ou réduire le développement des capabilités des patients.

Comme représenté sur la figure 5 , les situations dégradées sont particulièrement prégnantes au $\mathrm{CHU}$ où elles représentent $78 \%$ des séances observées (soit 98 séances sur 126), contre $32 \%$ au CLCC (soit 35 séances sur 110). 
Figure 5. Répartition des situations standards et des situations dégradées.

Figure 5. Distribution of standard and degraded situations

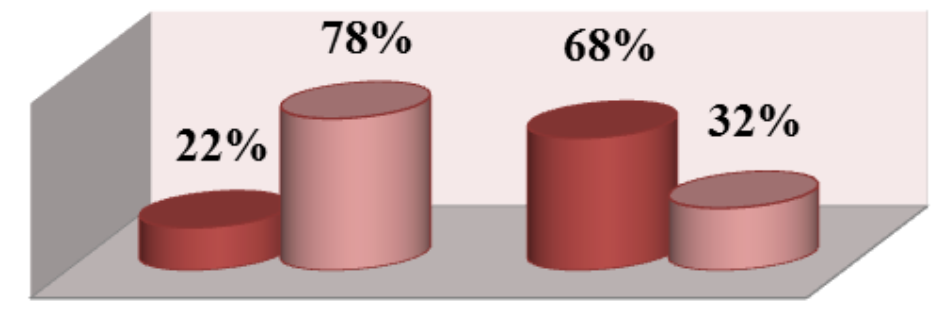

CHU CLCC

\section{$\square$ Situations standards $\square$ Situations dégradées}

\subsubsection{Les causes des retards}

Au total, 88 causes de retard ont été relevées au CHU sur les 126 séances de traitement observées, contre 47 au CLCC sur 110 séances de traitement observées. Elles peuvent être internes ou externes à l'organisation.

Les causes externes représentent $14 \%$ des situations au CHU et $28 \%$ au CLCC. Elles correspondent aux patients qui arrivent en retard, aux patients qui se trompent d'horaires, aux retards des ambulanciers et aux retards du service de chimiothérapie.

Les causes internes sont majoritaires et expliquent $86 \%$ des retards au CHU et $72 \%$ au CLCC. Les problèmes techniques liés à des pannes ou des dysfonctionnements de l'appareil de traitement représentent $10 \%$ des retards au CHU et $4 \%$ au CLCC. Mais la majorité des retards s'explique par le fait que la durée réelle de la séance de traitement est supérieure à la durée prescrite (91\% au CHU et $72 \%$ au CLCC). Les causes de cette augmentation sont de trois ordres (voir figure $\mathrm{n}^{\circ} 6$ ) :

1. Tâches supplémentaires à réaliser en plus de la délivrance du traitement comme la réalisation d'images portales permettant le contrôle du positionnement ou la réalisation de la dosimétrie in vivo permettant le contrôle de la dose d'irradiation reçue au cours de la séance de traitement (77\% au CHU et $68 \%$ au CLCC).

2. Durée prescrite de la séance (comme indiquée sur le planning) inférieure à la durée nécessaire pour la réalisation de la séance ( $16 \%$ au CHU et $18 \%$ au CLCC).

3. Difficulté à reproduire le positionnement attendu (7 \% au CHU et $14 \%$ au CLCC). 
Figure 6. Les causes de l'augmentation de la durée des séances de traitement. Figure 6 . The causes of the increase in the duration of treatment sessions

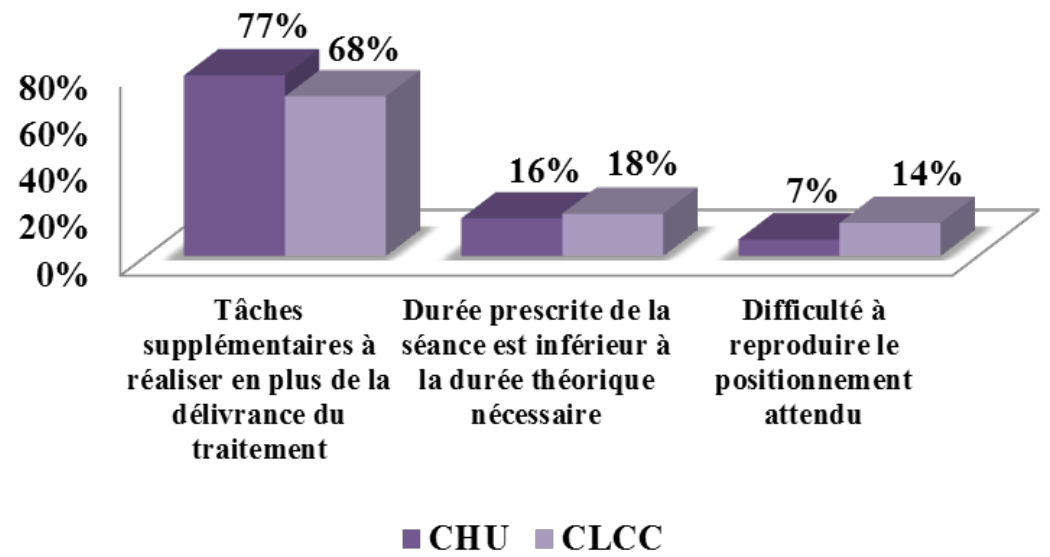

\subsubsection{Conséquence principale des situations dégradées : la mise à mal du care}

Tous les manipulateurs relatent qu'en situation de retard (situation dégradée), ils n'ont pas le temps de se consacrer à la relation. L'essentiel de leur activité consiste à accentuer la cadence de travail afin de rattraper le retard accumulé et de traiter tous les patients prévus en limitant le plus possible le temps passé par les patients en salle d'attente.

9 Leur travail est ainsi plus centré sur l'acte technique ou cure, par peur de commettre un oubli ou une erreur, au détriment du relationnel avec les patients. Ces situations sont en opposition aux valeurs de métier des manipulateurs puisqu'elles réduisent la pratique du care et ainsi la relation au patient.

o Les situations dégradées entrainent donc une diminution du temps des séances de traitement et ainsi une diminution des échanges manipulateur-patient. Elles mettent à mal la pratique du care puisqu'elles entrainent des difficultés à construire une relation de confiance ou à maintenir une continuité dans la relation.

Le tableau 2 fait la comparaison entre les situations standards et les situations dégradées. Les chiffres issus de ce tableau correspondent à la fréquence d'apparition des modes opératoires mis en œuvre sur l'ensemble des séances en situation standard et en situation dégradée, à partir d'une analyse des communications verbales lors des séances de traitement. 
Tableau 2. Fréquence d'apparition des modes opératoires mis en œuvre pour garantir chacune des dimensions du care en situation standard (SS) et en situation dégradée (SD) dans les deux établissements de soins.

Tableau 2. Frequency of occurrence of the procedures implemented to ensure each of the dimensions of care in standard situations (SS) and degraded situations (DS) in the two healthcare facilities

\begin{tabular}{|c|c|c|c|c|c|}
\hline \multirow[b]{2}{*}{$\begin{array}{l}\text { Les dimensions du } \\
\text { care }\end{array}$} & \multirow{2}{*}{$\begin{array}{c}\text { Les modes opératoires mis en } \\
\text { œuvre par les manipulateurs } \\
\text { pour garantir chacune des } \\
\text { dimensions }\end{array}$} & \multicolumn{2}{|c|}{ CHU } & \multicolumn{2}{|c|}{ CLCC } \\
\hline & & SS & SD & SS & SD \\
\hline \multirow[b]{2}{*}{$\begin{array}{l}\text { Personnalisation } d u \\
\text { soin }\end{array}$} & $\begin{array}{l}\text { Reconnaissance et respect du } \\
\text { patient en l'appelant par son nom } \\
\text { de famille }\end{array}$ & $12,5 \%$ & $3 \%$ & $100 \%$ & $100 \%$ \\
\hline & $\begin{array}{l}\text { Respect du patient par l'utilisation } \\
\text { de formules de politesse } \\
\text { (salutations en début et fin de } \\
\text { traitement) }\end{array}$ & $100 \%$ & $83 \%$ & $100 \%$ & $100 \%$ \\
\hline \multirow{3}{*}{ Veille attentive } & $\begin{array}{l}\text { Etre attentif à l'état émotionnel et } \\
\text { physique }\end{array}$ & $75 \%$ & $30 \%$ & $88 \%$ & $54 \%$ \\
\hline & Etre attentif aux effets secondaires & $25 \%$ & $5 \%$ & $35 \%$ & $6 \%$ \\
\hline & $\begin{array}{l}\text { S'intéresserà la vie du patient } \\
\text { (discussions relatives à la vie } \\
\text { privée) }\end{array}$ & $20 \%$ & $6 \%$ & $32 \%$ & $20 \%$ \\
\hline
\end{tabular}
deux structures. En effet, la reconnaissance et le respect du patient (emploi du nom de famille et de formules de politesse) sont systématiques au CLCC en situation standard et en situation dégradée. Au CHU en revanche, ils apparaissent dans seulement 12,5\% des séances en situation standard et $3 \%$ des séances en situation dégradée. L'appel du patient par son nom de famille est peu fréquent au $\mathrm{CHU}$, pourtant initialement perçu comme une action préventive de la part des manipulateurs. Quant au respect du patient par l'utilisation de formules de politesse, il apparaît dans $100 \%$ des séances en situation standard contre $83 \%$ en situation dégradée.

Pour faire face aux fréquents retards ( $78 \%$ de l'ensemble des séances de traitement observées au CHU contre $32 \%$ au CLCC), l'analyse de l'activité des manipulateurs du CHU en situation standard montre qu'ils anticipent un éventuel retard en accentuant leur cadence de travail. De ce fait, la pratique du care apparaît également mise à mal en situation standard.

94 Concernant la veille attentive, elle se voit affectée en situation dégradée. Les manipulateurs du CHU sont attentifs à l'état émotionnel et physique du patient dans $75 \%$ des séances en situation standard contre $30 \%$ des séances en situation dégradée. Les résultats vont dans le même sens au CLCC.

Ces résultats montrent bien que les dimensions du care sont mises à mal en situation dégradée et d'autant plus au $\mathrm{CHU}$ où ces situations sont les plus nombreuses. De plus, ils mettent en évidence l'impact du contexte, et plus particulièrement des conditions de travail sur la relation soignant-soigné et sur le développement des capabilités des patients. En effet, les situations dégradées entrainent une diminution des échanges manipulateur-patient et donc des difficultés dans la construction d'une relation de confiance, où tout du moins à avoir un suivi dans la relation. Les situations dégradées constituent donc des freins au développement de la coopération soignant-soigné puisqu'elles réduisent le temps d'échange nécessaire à la mise en œuvre de facteurs de 
conversion positifs. Nous pouvons donc dire que les professionnels ne sont pas toujours en situation capacitante pour pourvoir amener les patients à coopérer.

\section{Discussion}

\subsection{Promouvoir un environnement de soin capacitant pour favoriser le développement des capabilités des patients et des professionnels}

\section{individuelle du patient et
acteurs afin de permettre :}

- Au manipulateur d'allier l'aspect technique du soin (cure) et l'aspect relationnel du soin ( care), et avoir ainsi un sentiment de travail bien fait,

- Au patient de pouvoir être acteur dans la gestion de sa propre santé,

- Au collectif manipulateur-patient d'atteindre un objectif commun qui est la coproduction d'un soin sûr et efficace.

\subsection{Contributions de l'article sur l'approche des capabilités dans les organisations de soin}

Cette étude a permis d'approfondir l'approche des capabilités. En effet, au-delà de permettre la transformation d'une capacité en capabilité, les facteurs de conversion permettent également la création de ressources, ressources qui pourront aboutir au développement de nouvelles capabilités et donc d'augmenter le niveau de coopération. 
Les facteurs de conversion tels que la connaissance des risques et la motivation personnelle, permettent de créer une volonté de coopération chez les patients et ainsi d'orienter le choix de coopérer.

Les facteurs de conversion tels que la relation de confiance, l'encouragement et le renforcement positif, permettent également la création de ressources. La construction d'une relation de confiance permet aux manipulateurs d'établir une relation coopérative qui traduit un rapport de place coopératif et qui permet une certaine liberté d'expression. Par l'encouragement et le renforcement positif, le manipulateur assigne au patient une place d'acteur, de partenaire. Ils traduisent également d'un rapport de place visant la coopération du patient. Ils renforcent le fait que le patient a le droit de s'exprimer (liberté d'expression), ce qui peut amener à d'autres signalements.

2 L'absence de facteurs de conversion positifs peut être perçue comme un facteur de conversion négatif puisqu'elle peut entraver la transformation des capacités en capabilités et empêcher et/ou réduire la création de ressources. Le manque d'explication peut être également considéré comme un facteur de conversion négatif qui réduit le champ des possibles dans le développement des capabilités des patients.

3 Les situations dégradées sont un facteur de conversion négatif à part entière car elles empêchent les manipulateurs de pouvoir construire une relation de confiance, et ce faisant empêchent la création de nouvelles ressources nécessaires au développement de nouvelles capabilités. L'analyse de l'activité permet d'en comprendre les déterminants afin de prévenir leur apparition en mettant en place des actions efficaces. Par exemple, les analyses menées au sein des deux établissements ont montré que le roulement des manipulateurs sur l'ensemble des postes de traitement ne devait pas être trop rapide (inférieur à 3 mois). Cela permet de limiter le nombre de manipulateurs dans la prise en charge du patient tout au long de sa radiothérapie, et d'éviter une certaine méfiance des patients. En effet, certains patients qui sont pris en charge par des manipulateurs qu'ils n'ont pas l'habitude de voir ressentent une certaine méfiance qui est en totale opposition avec le développement d'une coopération basée sur la confiance. De la même manière, au cours d'une séance de radiothérapie, il est préférable que le manipulateur présent lors du positionnement du patient soit le même qui revient en salle en fin de traitement afin de permettre une certaine continuité dans la relation.

\subsection{La coopération des patients : une barrière de sécurité supplémentaire et un moyen de renforcer la satisfaction des soignants}

4 La coopération des patients ne peut être quelque chose que l'on exige d'eux : ce doit être une possibilité offerte, une capabilité potentielle, qui permette aux patients de se sentir acteurs à part entière de leur traitement, et plus généralement de leur propre santé. La présente recherche a mis en évidence le fait que la coopération du patient dans la sécurité et l'efficacité de son traitement constitue une barrière de sécurité supplémentaire qui mérite d'être encouragée afin d'améliorer les résultats en matière de sécurité des soins. Il est évident qu'elle ne peut en aucun cas remplacer les mesures de sécurité mises en place par les professionnels eux-mêmes dans leurs pratiques, et que la responsabilité en matière de sécurité incombe indiscutablement aux professionnels et 
aux établissements de soins. Il s'agit d'une voie de progrès qui, au-delà des objectifs d'efficacité et de sécurité des soins, participe à la santé des soignants.

La coopération des patients est rendue possible par la construction d'une relation de confiance (fondée sur le care). Cette relation au patient est au cœur du métier de soignant, ce qui donne sens au métier en lui-même. La majorité des manipulateurs expliquent que la relation au patient est ce qui donne sens à leur métier. Certains ont même choisi la radiothérapie plutôt que le diagnostic où la relation y est moindre. Favoriser la coopération des patients impacte donc favorablement la satisfaction des soignants et leur permet de redonner du sens à leur travail, sens qu'ils estiment perdre au fil des évolutions du système de santé. Pourtant dans les situations de soin, le patient est une référence qui va permettre de construire et de déterminer les identités des soignants ainsi que leurs systèmes de valeurs (Aïach, \& Fassin, 1994 ; Carricaburu, \& Ménoret, 2004). En effet, plus le professionnel de santé est proche du patient, plus il se sent valorisé et confirmé dans sa fonction, ce qui se traduit par un meilleur engagement des soignants dans leur travail.

\section{BIBLIOGRAPHIE}

Aïach, P., \& Fassin, D. (1994). Les métiers de la santé. Enjeux de pouvoir et quête de légitimité.

Paris : Anthropos.

Arnoud, J., \& Falzon, P. (2013). La co-analyse constructive des pratiques. In P. Falzon (Ed.), Ergonomie constructive. pp. 223-236. Paris : PUF.

ASN. (2009). La sécurité des traitements en radiothérapie externe : le point de vue des acteurs français. Contrôle, $\mathrm{n}^{\circ} 185$.

ASN. (2011a). Identification du patient. La sécurité du patient. Pour une dynamique de progrès, $\mathrm{n}$ -1 mars.

ASN. (2011b). La première séance « à blanc ». La sécurité du patient. Pour une dynamique de progrès, $\mathrm{n}^{\circ} 2$ novembre.

ASN. (2013). Quels événements à déclarer à l'ASN ? La sécurité du patient. Pour une dynamique de progrès, $\mathrm{n}^{\circ} 4$ avril.

Bibault, J.E., Pernet, A., Mollo, V., Gourdon, L., Martin, O., \& Giraud, P. (2016). Empowering patients for radiation therapy safety: Results of the EMPATHY study. Cancer/radiothérapie, 20(8), pp. 790-793.

Buetow, S., \& Elwyn, G. (2007). Patient safety and patient error. The Lancet, 369 (9556), pp. 158-161.

Carricaburu, D., \& Ménoret, M. (2004). Sociologie de la santé. Institutions, professions et maladie. Paris : Armand Colin.

Cerf, M., \& Falzon, P. (2005). Situations de service : travailler dans l'interaction. Paris : PUF. 
Conseil de l'Europe (2001). Le développement de structures permettant la participation des citoyens et des patients au processus décisionnel concernant les soins de santé. Strasbourg : Editions du Conseil de l'Europe.

Coulter, A., \& Jenkinson, C. (2005). European patients' views on the responsiveness of health systems and healthcare providers. European Journal of Public Health, 15(4), pp. 355-360.

Coulter, A., \& Ellins, J. (2006). Effectiveness of strategies for informing, educating, and involving patients. BMJ, 335(7609), pp. 24-7.

Davis, R.E., Jacklin, R., Sevdalis, N., \& Vincent, CA. (2007). Patient involvement in patient safety: what factors influence patient participation and engagement. Health Expect, 10(3), pp. 259-67.

Davis R.E., Sevdalis, N., \& Vincent C. (2011). Patient involvement in patient safety- How willing are patients to participate? BMJ, Quality and Safety, 20(1), pp. 108-114.

Doumont, D., \& Aujoulat, I. (2002). L'empowerment et l'éducation du patient. Série de dossiers techniques, Réf. 02-18.

Falzon, P. (2013). Pour une ergonomie constructive. In P. Falzon (Ed.), Ergonomie constructive (pp. 1-15). Paris : PUF.

Falzon, P., \& Mollo, V. (2009). Pour une ergonomie constructive : les conditions d'un travail capacitant. Laboreal, 5(1), pp. 61-69.

Fernagu-Oudet, S. (2012). Concevoir des environnements de travail capacitants : L'exemple d'un réseau réciproque d'échanges des savoirs. Formation-Emploi, 3(119), pp. 7-27.

Garza, C., de la \& Weill-Fassina, A. (2000). Régulations horizontales et verticales du risque. In T.H. Benchekroun \& A. Weill-Fassina, A. (Eds), Le travail collectif : perspectives actuelles en ergonomie (pp. 217-234). Toulouse: Octarès Éditions.

Guadagnoli, E., \& Ward, P. (1998). Patient participation in decision-making. Social Science \& Medicine, 47(3), pp. 329-339.

Hall, J. (2007). What are the roles for the patient in patient safety research? In Porto conference on Patient Safety Research - shaping the European agenda. Porto, Portugal : 24-26th September 2007.

Hesbeen, W. (1999). Le caring est-il prendre soin ? Perspective soignante, $n^{\circ}$ 4, pp. 1-20.

Hoc, J.M. (1996). Supervision et contrôle de processus : la cognition en situation dynamique. Aubenas: PUG.

Hollnagel, E. (2010). Prologue: The scope of resilience engineering. In E. Hollnagel, J. Pariès, D. Woods, \& J. Wreathall (Eds.), Resilience Engineering in Practice: A Guidebook. Ashgate: Studies in Resilience Engineering.

Jorm, M.C., Dunbar, N., Sudano, L., \& Travaglia, J.F. (2009). Should patient safety be more patient centred? Australian Health Review, 33(3), pp. 390-399.

Kohn, L., Corrigan, J., \& Donaldson, M. (1999). To error is human: building a safer healthcare system. Washington DC: National Academy Press.

Koutantji, M., Davis, R., Vincent, C., \& Coulter, A. (2005). The patient's role in patient safety: engaging patients, their representatives, and health professionals. Clinical risk, 11(3), pp. 99-104.

Lau, D.H. (2002). Patient empowerment: a patient-centred approach to improve care. Hong Kong Med J, 8(5), pp. 372-374. 
Lyons, M. (2007). Should patients have a role in patient safety? A safety engineering view. Quality and Safety Health Care, 16(2), pp. 140-142.

Martin, H.M., Navne, L.E., \& Lipczak, H. (2013). Involvement of patients with cancer in patient safety: a qualitative study of current practices, potentials and barriers. BMJ Quality and Safety 22 (10), pp. 836-842.

Mollo, V., \& Falzon, P. (2004). Auto- and allo-confrontation as tools for reflective activities. Applied Ergonomics, 35(6), pp. 531-540.

Mollo, V., \& Falzon, P. (2009). Le corps comme objet de l'interaction médecin-patient. Corps, 9, pp. 69-75.

Mollo, V., Pernet, A., Moutel, G., Duchange, N., \& Giraud, P. (2011). Le patient peut-il et doit-il participer à la gestion des risques en radiothérapie ? Cancer/radiothérapie, 15(3), pp. 176-181.

Neboit, M., Cuny, X., Fadier, E., \& Ho, M.T. (1990). Fiabilité humaine : présentation du domaine. In J. Leplat \& G. De Terssac (Eds.), Les facteurs humains de la fiabilité dans les systèmes complexes (pp. 23-46). Toulouse : Octarès Éditions.

Pernet, A. (2010). Co-construire la sécurité des soins : La participation des patients à la sécurité des soins en radiothérapie. Mémoire de Master Sciences du Travail et de la Société, mention Ergonomie, spécialité Recherche. CNAM, Paris.

Pernet, A. (2013). Coproduire un soin sûr et efficace : le développement des capabilités des patients en radiothérapie. Thèse de Doctorat en Ergonomie, Conservatoire National des Arts et Métiers, Paris.

Pernet, A., Mollo, V., \& Giraud, P. (2012). La participation des patients à la sécurité des soins en radiothérapie une réalité à développer. Bulletin du Cancer, 99(5), pp. 581-7.

Pernet, A., Mollo, V., Bibault, J.E., \& Giraud, P. (2016). Evaluation of patients' engagement in radiation therapy safety. Cancer/radiothérapie, 20(8), pp. 765-767.

Schmidt, K. (1991) Cooperative work, a conceptual framework. In J. Rasmussen, J Leplat, B. Brehmer (eds), Distributed decision making, cognitive models for cooperative work, pp. 75-110.

Sen, A. (1999). Development as freedom. Oxford: Oxford University Press.

Sen, A. (2009). The idea of justice. Cambridge: Harvard University Press.

Six-Touchard, B., \& Falzon, P. (2013). L'auto-analyse du travail : un outil réflexif pour le développement des compétences. In P. Falzon (Ed.), Ergonomie constructive (pp. 237-249). Paris: PUF.

Unruh, KT., \& Pratt, W. (2007). Patients as actors: the patient's role in detecting, preventing, and recovering from medical errors. In J Med Inform, 76(Suppl 1), pp. 236-44.

Van Wijk, G. (2000) : Confiance et Structure. In R. Laufer, \& M. Orillard (Eds.), La confiance en question. pp. 263-280. Paris : Harmattan.

Vincent, C., \& Coulter, A. (2002). Patient safety: what about the patient? Quality and Safety in Health Care, 11(1), pp. 76-80.

Vincent, C., \& Davis R.E. (2012). Patient and families as safety experts. CMAJ, 184(1), pp. 15-16.

Waterman, A.D., et al. (2006). Patient Involvement in Error Prevention. In Brief Report: Hospitalized Patients « Attitudes About and Participation in Error Prevention, pp. 367-370.

Weingart, S.N., et al. (2004). Lessons from a patient partnership intervention to prevent adverse drug events. International Journal for Quality in Health Care, 16(6), pp. 499-507. 
Weingart, S.N. et al. (2005). What can hospitalize patients tell us about adverse events? Learning from patient-reported incidents. In Gen Intern Med 20(9), pp. 830-6.

WHO. (2013). Exploring patient participation in reducing health-care-related safety risks. WHO Regional Office for Europe.

\section{ANNEXES}

\section{Situation 5 (Codage: R5/C2/CT2)}

\begin{tabular}{|l|l|}
\hline Éléments du contexte & $\begin{array}{l}\text { Caractéristique du patient réalisant sa séance de } \\
\text { traitement }\end{array}$ \\
\hline Accélérateur : & Sexe $: \mathrm{F} \square \mathrm{M}$ 囚 \\
\hline Date : & Age $: 78$ ans \\
\hline Équipe : & $\mathrm{N}^{\circ}$ de la séance $: 18^{\text {ème }}$ \\
\hline $\begin{array}{l}\text { Horaire prescrit : } \\
\text { 18h10-18h20 } \\
\text { Horaire réel }: 18 \mathrm{~h} 30-18 \mathrm{~h} 36\end{array}$ & Type de cancer : Médiastin (Bronches) \\
\hline
\end{tabular}

\begin{tabular}{|l|l|}
\hline En salle de traitement & Communications verbales \\
\hline & Patient : Bonjour \\
Manip : Bonjour Monsieur X (X = nom de \\
$\begin{array}{l}\text { Préparation de la table de traitement (contentions et } \\
\text { famille du patient) }\end{array}$ \\
Patient : On est en retard aujourd'hui \\
Manip : Oh oui
\end{tabular}




\begin{tabular}{|c|c|}
\hline $\begin{array}{l}\text { Positionnement du patient } \\
\text { (+ table et accélérateur) / Vérifications à l'aide des } \\
\text { points de tatouages ou marquages + écran en salle }\end{array}$ & $\begin{array}{l}\text { Patient: Il n'est pas bon celui-là (cale- } \\
\text { tête) } \\
\text { Manip : Effectivement ce n'est pas le bon } \\
\text { Manip : Vous avez raison de vous } \\
\text { permettre de me le dire } \\
\text { Patient : Je vous aime bien vous } \\
\text { Manip : J'espère bien (sourire) } \\
\text { Manip : Allez à tout de suite, d'ici } \\
4 \text { minutes } \\
\text { Patient : Ok } \\
\text { Manip : Banco }\end{array}$ \\
\hline Traitement & Communications verbales \\
\hline En salle de traitement & Communications verbales \\
\hline Aide le patient à descendre & $\begin{array}{l}\text { Vous pouvez lâcher les bras monsieur } \mathrm{X} \\
(\mathrm{X}=\text { nom de famille du patient }) \\
\text { Patient : Merci }\end{array}$ \\
\hline Nettoyage de la table de traitement & $\begin{array}{l}\text { Patient : Allez à demain } 17 \mathrm{~h} 30 \\
\text { Manip : Bonne soirée }\end{array}$ \\
\hline
\end{tabular}

\section{RÉSUMÉS}

Cet article cherche à comprendre la dynamique constructive des capabilités des patients en radiothérapie. Dans cette étude, les capabilités des patients renvoient à ce qu'ils sont réellement en mesure de faire en termes de sécurité et d'efficacité des soins. Plus particulièrement, nous allons mettre en évidence les facteurs de conversion qui favorisent ou entravent le développement des capabilités des patients, c'est-à-dire les facteurs qui permettent leur coopération. La méthodologie a combiné des observations de séances de traitement ainsi que des entretiens auprès de professionnels de radiothérapie et de patients. Les principaux résultats soulignent le rôle majeur de la connaissance des risques associés aux soins, de la motivation personnelle, de la relation de confiance, de l'encouragement et du renforcement positif dans le développement des capabilités des patients. De plus, ils permettent de voir comment le contexte et plus précisément les conditions de travail peuvent impacter sur le développement des capabilités des patients et donc sur la coopération soignant-soigné. La discussion met l'accent sur le fait que le développement des capabilités des professionnels est le point de départ au développement des capabilités des patients.

This article seeks to understand the constructive dynamics of patients' capabilities in radiotherapy. In this study, "patients' capabilities" refers to what they are actually able to do in terms of safe and effective care. In particular, we highlight the conversion factors that promote or hinder the development of patients' capabilities, that is to say, the factors that enable their 
cooperation. The methodology combined observations of treatment sessions and interviews with radiotherapy professionals and patients. The main results highlight the key role that knowledge of risks associated with care, personnel motivation, trust relationships, encouragement and positive strengthening play in the development of patients' capabilities. In addition, they show how context and, more specifically, working conditions may impact the development of patients' capabilities and therefore caregiver-patient cooperation. The discussion focuses on the development of professionals' capabilities as the starting point and the condition for the development of patients' capabilities.

INDEX

Keywords : patient capabilities, patient participation, patient safety, radiotherapy Mots-clés : capabilités des patients, participation des patients, sécurité des patients, radiothérapie

\section{AUTEURS}

\section{ADELINE PERNET}

Centre d'Étude et de Recherche : Travail, Organisation, Pouvoir (CERTOP, UMR5044), Maison de la recherche, 5 allées Antonio Machado, 31058 Toulouse Cedex 9 pernet.adeline@gmail.com

\section{VANINA MOLLO}

IPST-Cnam, 118 route de Narbonne, 31062 Toulouse

Centre d'Étude et de Recherche : Travail, Organisation, Pouvoir (CERTOP, UMR5044), Maison de la recherche, 5 allées Antonio Machado, 31058 Toulouse Cedex 9

vanina.mollo@ipst-cnam.fr

\section{PIERRE FALZON}

Cnam, Centre de Recherche sur le Travail et le Développement (CRTD), 41 rue Gay-Lussac, 75005

Paris

pierre.falzon@cnam.fr 